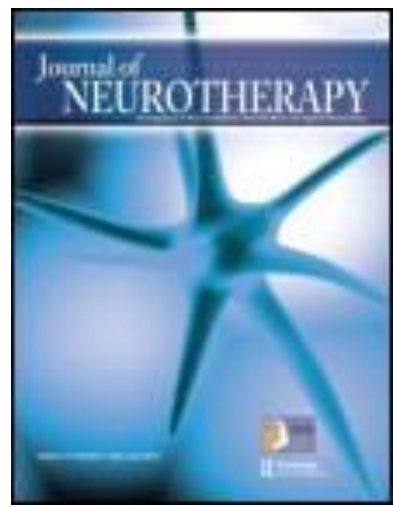

Journal of Neurotherapy: Investigations in Neuromodulation, Neurofeedback and Applied Neuroscience

\title{
The Improvement of Severe Psychomotor and Neurological Dysfunctions Treated with the Tomatis Audio-Psycho-Phonology Method Measured with EEG Brain Map and Auditory Evoked Potentials
}

\author{
J. Vervoort ${ }^{a}$, M. J. A. de Voigt ${ }^{b} \&$ W. Van den Bergh MD ${ }^{c}$ \\ ${ }^{a}$ Atlantis-vzw Institute and Mozart-Brain-Lab , Sint-Truiden, Belgium \\ ${ }^{\mathrm{b}}$ Mozart-Brain-Lab and the Atlantis-vzw Institute, Sint-Truiden, Belgium \\ ${ }^{c}$ Mozart-Brain-Lab and the Atlantis-vzw Institute, Sint-Truiden, Belgium \\ Published online: 08 Sep 2008.
}

To cite this article: J. Vervoort , M. J. A. de Voigt \& W. Van den Bergh MD (2008) The Improvement of Severe Psychomotor and Neurological Dysfunctions Treated with the Tomatis Audio-Psycho-Phonology Method Measured with EEG Brain Map and Auditory Evoked Potentials, Journal of Neurotherapy: Investigations in Neuromodulation, Neurofeedback and Applied Neuroscience, 11:4, 37-49, DOI: $\underline{10.1080 / 10874200802169621}$

To link to this article: http://dx.doi.org/10.1080/10874200802169621

PLEASE SCROLL DOWN FOR ARTICLE

( ) International Society for Neurofeedback and Research (ISNR), all rights reserved. This article (the "Article") may be accessed online from ISNR at no charge. The Article may be viewed online, stored in electronic or physical form, or archived for research, teaching, and private study purposes. The Article may be archived in public libraries or university libraries at the direction of said public library or university library. Any other reproduction of the Article for redistribution, sale, resale, loan, sublicensing, systematic supply, or other distribution, including both physical and electronic reproduction for such purposes, is expressly forbidden. Preparing or reproducing derivative works of this article is expressly forbidden. ISNR makes no representation or warranty as to the accuracy or completeness of any content in the Article. From 1995 to 2013 the Journal of Neurotherapy was the official publication of ISNR (www. Isnr.org); on April 27, 2016 ISNR acquired the journal from Taylor \& Francis Group, LLC. In 2014, ISNR established its official open-access journal NeuroRegulation (ISSN: 2373-0587; www.neuroregulation.org).

THIS OPEN-ACCESS CONTENT MADE POSSIBLE BY THESE GENEROUS SPONSORS

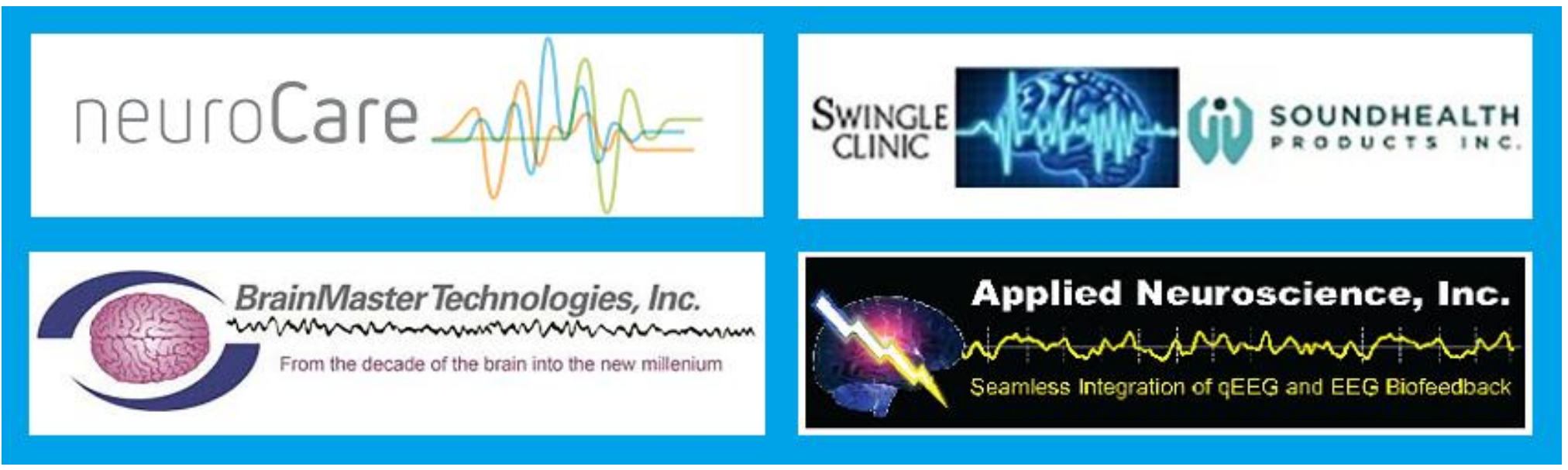




\title{
The Improvement of Severe Psychomotor and Neurological Dysfunctions Treated with the Tomatis Audio-Psycho-Phonology Method Measured with EEG Brain Map and Auditory Evoked Potentials
}

\author{
J. Vervoort \\ M. J. A. de Voigt \\ W. Van den Bergh, MD
}

\begin{abstract}
Background. Pioneering research of Tomatis led to the introduction of the Audio-Psycho-Phonology (APP), to treat, improve, or remediate severely neurologically impaired individual.

Method. Here we present for the first time independent data on the APP method with auditive evoked potentials and electroencephalographically based brain mapping.

Results. The improvement of these 4 patients by undergoing APP treatment is shown in their listening curves as well as in the results of the EEG based brain maps and the auditory evoked potentials.

Conclusion. 1. APP method seems to be an effective and harmless treatment for patients with severe psychomotor and/or neurological dysfunctions.

2. Auditory evoked potentials and electroencephalographically based brain mapping seems to be an appropriate examination to demonstrate neurological changes after APP therapy.

Besides the presentation of the four cases, a short overview of the method is presented.
\end{abstract}

KEYWORDS. Audio-Psycho-Phonology, auditory evoked potentials, EEG based brain mapping, neurological deficits, Tomatis

The Tomatis Audio-Psycho-Phonology (APP) listening to specially processed and filtered listening therapy trains the individual's music, a mother's voice, or their own voice. neurological and physiological systems by This training is based on the fact that the

J. Vervoort is a retired special education teacher and the founder and leader of Atlantis-vzw Institute and Mozart-Brain-Lab, Sint-Truiden, Belgium.

M. J. A. de Voigt, retired professor of physics, Tech. Univ. Eindhoven and Tomatis therapist APP Listening Center Gorinchem, The Netherlands, is affiliated with the Mozart-Brain-Lab and the Atlantis-vzw Institute, SintTruiden, Belgium.

W. Van den Bergh, Neurologist, private practice, Leuven, Belgium, is affiliated with the Mozart-Brain-Lab and the Atlantis-vzw Institute, Sint-Truiden, Belgium.

Address correspondence to: M. J. A. de Voigt, Center of Listening Therapy, Kriekenmarkt 25, 4201 AN Gorinchem, The Netherlands (E-mail: m.j.a.de.voigt@tue.nl).

\footnotetext{
Journal of Neurotherapy, Vol. 11(4) 2007

Copyright (c) 2007 ISNR. All rights reserved. doi: $10.1080 / 10874200802169621$
} 
ear is the most sensitive entrance to the central neurological system (i.e., a large part of the brain, the vestibule-cochlear, the vagus and recurrent nerves). The sounds, after filtering, are presented to the two ears by a headphone as well as to the skull by a bone vibrator. In addition to frequency filtering, the various intensities and delays between those components are also adjusted. The processed sounds stem mainly from Mozart concerts and Gregorian chants. Tomatis has developed specific electronics during the past 50 years, which are utilized and further elaborated at Mozart Brain Lab (MBL) in Sint-Truiden, Belgium. Professor Tomatis, a medical specialist in hearing, developed the method and has conducted most of the research to date. He discovered the great psychological and physical influence the ear has on our functioning, communication, learning, language, and speech (Madaule, 1994; Tomatis, 1972, 1989, 1991). His research over many experiments yielded a device called the "Electronic Ear," which has been successfully applied in therapies all over the world treating more than 25,000 people during the last 30 years, of which about 5,000 were treated in Sint-Truiden. Statistics show long-lasting improvements in about $80 \%$ of those treated as obtained from investigations in many institutions; the results are summarized at http://www.tomatis.com.

The replication of the method has been accomplished with groups of children at schools, including placebo groups (Schydlo, 2002; Tomatis, 1991). Independent replication is possible since the introduction, about 12 years ago, of electroencephalographic- (EEG-) based brain mapping at the Atlantis Institute in St.-Truiden, in combination with the Tomatis listening therapy. From the wealth of data available at SintTruiden we are presenting only four cases here to illustrate the replication method.

In general, changes because of the therapy are very visible in the measured EEG-brain maps and in the AEPs. They can be correlated with changes in the listening tests and with the observed improvements in the problems of the individual participants. Out of the many thousands of available files, we have selected four participants, each with severe problems. The problems of each participant were reported very difficult or impossible to remediate by common medical or therapeutic methods. The data and background of two participants are described in detail, and two other participants are described more briefly, but all achieved remarkable results. This article ends with general conclusions and recommendations for the treatment of certain pathological cases.

The APP, introduced by Alfred Tomatis, is described extensively elsewhere (Madaule, 1994; Tomatis, 1972, 1989, 1991) along with the history and motivation of its development beginning in 1947 (Tomatis, 1991). Here we summarize the main ingredients of the method, without too much discussion of the underlying arguments. It is a wellknown phenomenon that sounds do stimulate our total neurological and physiological system, and in particular hearing organs and the brain with the coordination between the two hemispheres. Also the psychological effects of sound and music are well experienced. Tomatis studied the physiological development of the ear from the first stage of pregnancy forward (e.g., Tomatis, 1981). The many experiments with pregnant women revealed that the fetus reacts to sounds, particularly above $8000 \mathrm{~Hz}$. It appeared that the high tones are transmitted dominantly via the spine and the pelvis of the mother to the body of the fetus, thus via bone conduction. This evidence, and also the high tonus of the muscles directly after birth, indicates the effectiveness of high tones in stimulating the neural system and muscles. The hearing organs of the fetus are fully developed after about the 4th month of pregnancy, in contrast to the rest of the neural system that takes more than 20 years to be fully myelinated. The part of the cochlea near the base, sensitive for the high frequencies, is developed first and is also the most sensitive part. Those observations led Tomatis to acknowledge the central role of the ear in our functioning. Further experiments with singers revealed to Tomatis the strong correlation between hearing and the voice. This enabled him to remediate the voice 
(e.g., false singing of incorrect tones) using a listening therapy. Those findings and many other experiences led Tomatis to formulate three important statements (Tomatis, 1991):

- The voice contains only those frequencies the ear can perceive.

- If we give the ear the possibility to hear correctly, we improve instantaneously and unconsciously the vocal utterance.

- It is possible to change the phonation with an auditive stimulation during a certain time.

From various experiments Tomatis discovered that high tones, particularly above $8000 \mathrm{~Hz}$, are perceived well by the fetus, and he derived the hypothesis that they cause efficient cortical stimulation (Tomatis, 1981, 1991). Therefore Tomatis called the ear "the battery for the brain." He distinguished three main frequency regions and related functions, with the notion that the cochlea analyzes all frequencies, particularly high tones, and that the vestibule perceives also low tones. The low region up to about $1000 \mathrm{~Hz}$ is predominantly related to equilibrium, motor, and vegetative functions. Those tones perceived by the vestibule resonate predominantly on the body. The middle region, of about 1000 to $3000 \mathrm{~Hz}$, is related to speech, language, and communication, because this frequency band is strongly represented in the voice. The region of high tones above $3000 \mathrm{~Hz}$ relates more to associative and thinking processes, because the highest frequencies resonate predominantly in the head. He also emphasized bone conduction with respect to the air conduction. Bone conduction dominates in the case of hearing our own voice, thus psychologically it relates to the communication with ourselves. Air conduction via the ears, however, dominates in the case of hearing external sounds, thus it relates to communication with those in our surroundings. Harmony between the two forms of conduction is important for a proper balance between our inner and the outside world.

The sounds cause both physiological and neurological stimulation. The first type of stimulation is because of the movements of the ossicles and of the membranes (i.e., the eardrum and the oval and circular membranes, thereby training the relevant muscles of the middle ear). The neurological stimulation concerns among other things the hearing, equilibrium, vagus nerve, recurrent nerves, and the brain. The recurrent nerve for the left ear has a longer pathway to the larynx than for the right ear, which, combined with the localization of the Broca motor center of speech only in the left hemisphere, results in a retardation of speech by about $0.03 \mathrm{sec}$ when the feedback is dominated by the left ear (Tomatis, 1991). This fact and the dominance of the left hemisphere for logic, abstract thinking, language, reading, writing, and calculus stimulated Tomatis to emphasize training of the right ear. The neural stimulation by sound of all organs and muscles of the body is largely accomplished by means of the vagus nerve, which branches from the eardrum and from the outer hearing channel via the spinal marrow to all those peripheries in the body.

The starting point of the therapy is the listening test, which consists of an audiogram, interpreted both physically and psychologically by a trained APP consultant. An ideal curve is given in Figure 1, which according to Tomatis reaches a maximum sensitivity in the communication (speech) region of 1000 to $3000 \mathrm{~Hz}$. This test indicates possible

FIGURE 1. The listening test with ideal curve according to Tomatis. Note. The blue curve represents the air conduction and the red one the bone conduction. Intermediate frequencies at 750, 1500, 3000 , and $6000 \mathrm{~Hz}$ are omitted for clarity.

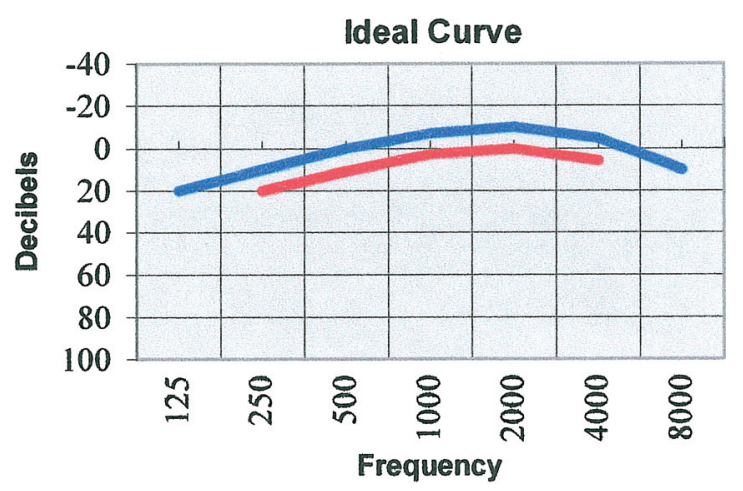


problems in certain frequency regions in bone and/or air conduction for the left or right ear, thus consequently the right or left hemisphere, respectively. The latter phenomenon is based on the notion that contralateral connections between the ear and the hemispheres are stronger than the ipsi-lateral ones. A high bone conduction with respect to the air conduction signifies that the participant is dominantly listening to himself or herself, thus living in his or her own world and controlling poorly the communication with his or her surrounding. Localization errors are registered if the wrong ear perceives the sounds. This may signify confusion in orientation and between emotion and rationality. The discrimination between tones, or selectivity, is measured between adjacent frequencies in the listening test. A closed selectivity signifies screening from the outside as a kind of protection. The ear dominance, or laterality, is measured as an indication that the participant is more or less emotionally or rationally oriented.

Based on the results of the listening test, an individualized listening therapy plan is constructed. This includes, in most cases, certain filtered music, the mother's voice, and the individual's own voice but always a processing whereby a continuous, but irregular, alteration between filtered low and high tones is provided. The basic sound material is that of Mozart's concerts, which are known for their great harmony, high rhythm, large density of high frequencies, and universality. Therefore they provide great cortical stimulation. To provide sufficient training of the body coordination Gregorian chants are used because of the low rhythm and quietness. The therapy starts with a passive phase of unfiltered music, followed by highly filtered and again unfiltered music, on both the ears and skull. Particularly for young persons the mother's voice is also recorded and used in the highfilter situation. The therapy is concluded by an active phase using the client's own voice with reading and singing. The therapy is executed uniquely in qualified centers with specially developed electronics. Generally, the sounds for the listening therapy are provided in cycles of 5 to 9 days with 2 to
$4.5 \mathrm{hr}$ of listening and with two interruptions of $1.5 \mathrm{hr}$ in the latter case. At the beginning of each cycle, a Listening test is conducted to check the changes and to adapt the program. Between the cycles intermissions of at least 4 weeks are planned. The total duration of the therapy depends on the participant's problems and response to treatment but consists in most cases of at least three cycles.

\section{METHOD}

\section{The EEG-Brain Mapping}

Many independent neurological investigations indicated that several problems related to language and communication, such as dyslexia, speech, and aphasia, are correlated with the pathology of the left temporal hemisphere (Mason \& Mellor, 1984; Pinkerton, Watson, \& McClelland, 1989; Tallal, Miller, \& Fitch, 1993; Tallal et al., 1996). Relief of those problems was favorably accomplished with auditory stimulation, particularly of the right ear (Merzenich et al., 1996; Tallal et al., 1996). In recent years, auditory-evoked response techniques have been utilized to objectively assess integrity of the central auditory system in children with learning disabilities, autism, and language and attention deficit disorders (Van den Bergh, 1998).

Auditory evoked potentials (AEPs) are measured at the Atlantis Institute with 20 to 30 electrodes on the skull using the International 10-20 System of Electrode Placement. Auditory clicks or tones are presented mostly into the left ear and the measurements are registered (Van den Bergh 1998). The responses are recorded as functions of time in the millisecond regime. In the time domain, the first $10 \mathrm{msec}$ relate to functions of the lower brain stem and are an objective measure of peripheral hearing in very young or uncooperative children. The middle latency $(10-50 \mathrm{msec})$, the long latency $(50-100 \mathrm{msec})$, and the cognitive auditory potentials $(100-700 \mathrm{msec})$ relate to functions of the higher brain stem, the temporal primary cortex, and to the frontal association cortex, respectively. Deviation 
of the middle latency potentials from normal patterns is indicative for problems with consciousness and attention mechanisms and for the sampling time of the central auditory system. Language development disorders show up as deviations in the long latency auditory potentials, particularly in the temporal complexes T4 (right) and T3 (left).

With the cognitive auditory potentials, the mental processing mechanisms of the auditory perception (attention mechanisms) are explored. There are 150 tones presented to the participant via headphones. There are 120 tones that are frequent, "standard," and low pitched, and 30 are "rare," high-pitched tones. This test is performed under both the attention (to the rare tones) and nonattention condition. Most important is to measure the different amplitudes (e.g., the one of N200) under those two conditions for a certain individual. Therefore relative amplitude scales are sufficient, particularly when later the measurements are repeated for the same individual with the same adjustments.

An electrical negative response appears $100 \mathrm{msec}$ after the tones (N100) at which time the participant's attention is directed to the task. The N100 intensity should increase as a function of early selective attention. Two hundred msec after the rare tones, an electrical negative response appears (N200), even when the participant is not attending. This is a measure of automatic discrimination. Three hundred msec after the rare tones, in the attention condition, an electrical positive response appears (P300) and is a measure for controlled meaningful stimulus processing.

Between 500 and $1000 \mathrm{msec}$ after the tones in the attention condition, an electrical negative response appears in the frontal brain areas (late frontal negativity). This is a measure for additional controlled processing of the attending stimuli.

Those cognitive potentials are in many cases disturbed in children with cognitive immaturity, attention deficit disorders, and learning disabilities.

The QEEG data are taken with the same equipment (Sirius, ESAOTE BIOMEDICA). They are processed quantitatively, in contrast to the classical EEG, as to reconstruct a map of responses over the brain surface, called the brain map. The standard "only eyes closed condition" was being used. Neither database nor statistical analysis was used because of the comparison of each case with itself (before and after treatment), which provides a relative indication of the progress. Standard QEEG algorithms were used both in the measurements and analysis. In the frequency domain one usually distinguishes four different regions, that is, Delta $(\delta=0-4 \mathrm{c} / \mathrm{sec})$, Theta $(\theta=4-7 \mathrm{c} / \mathrm{sec})$, Alpha $(\alpha=8-12 \mathrm{c} / \mathrm{sec})$, and Beta $(\beta=12-30 \mathrm{c} / \mathrm{sec})$. The brain maps can be constructed for those four frequency regions. For instance, in the case of a poor alertness one observes little $\alpha$ and $\beta$ activity and/or much $\delta$ and $\theta$ activity. For learning and language the activities in the left temporal hemisphere are particularly interesting.

\section{RESULTS}

We present the data from four individual participants, each exhibiting pathological findings. We verified that no other therapies or treatments were being provided to these four participants during the listening therapy treatment period. Only Participants 2 and 4 had continued to receive some medication after treatment.

\section{Case 1: Lena}

Lena had a history of retardation in general psychomotor and speech development. She had no conscious contact to the environment, had no orientation with other persons, had autistic tendencies, lived as if in a cocoon, and exhibited extensive retardation of fine and gross motor skills. The pregnancy of the mother was very troubled, with loss of amniotic fluid, bleeding, hospitalization, and medication (e.g., antibiotics). The birth, however, was normal and at the correct gestational time. At an age of 3.5 months Lena was enclosed in a spread pant, to force her to lie on her back for 4 month, thus retarding her early development even more. During the last months of pregnancy the sound transfer of 
the mother's voice had been far from ideal, because of the diminished amniotic fluid and the enforced laying and resting of the mother. The antibiotics influenced negatively the development of the child. The voice of the anxious mother also changed to the low tones, because she was afraid of losing her child.

At Lena's age 2, a listening therapy started at Atlantis, obviously without listening test; thus therapy was based on the experience of the therapist. The parents showed considerable dedication until Lena was 7 years old with a total of 31 cycles of 5 days, on the average six cycles per year. The first cycle was 12 days with unfiltered Mozart and Gregorian music. Thereafter cycles followed at about 6-week intervals, with the mother's voice filtered at $8000 \mathrm{~Hz}$, achieving a psychological return to the prenatal phase. During this phase, the participant hears the tones like a fetus in the prenatal phase might have heard, thus roughly above $8000 \mathrm{~Hz}$ according to experiments by Tomatis, previously discussed. Slow progress was noticed, first in the vestibule domain (i.e., small move- ments, thereafter some eye contact and even some alterations between aggression and affection toward the mother). A year later some development progress had occurred: She showed better contact with the external environment. At age 4, the first brain map was obtained (see Figure 2a). This already showed a reasonable ground rhythm in the $\theta$ range $(4.0-7.5 \mathrm{~Hz})$, but almost no activity in the $\alpha$ and $\beta$ domains, thus little alertness. In the cognitive-auditory cortex activity, a very weak auditory AEP N200 signal (evoked in an auditory oddball paradigm) indicated poor automatic stimulus discrimination.

Lena listened to her mother's voice and had been provided with a motor tuning of the Electronic Ear to stimulate simultaneously and harmonically the motor and auditory cortex, by exciting particularly the vestibule with low tones and the cochlea with high tones At age 5, the clinician succeeded for the first time in obtaining a listening test (see Figure 3).

The high bone conduction (red line) is very pronounced, characteristic for children living in their own world. The disorder

FIGURE 2. Brain maps of Lena during (a) and after the therapy (b). Note. The four maps represent the $\delta, \theta, \alpha$, and $\beta$ waves with indicated frequencies. Note that the different scales (vertical color bars), which are slightly larger in case B than in A, indicate relative brain activity (deduced from the electrode voltages) with red high and blue low brain activity.

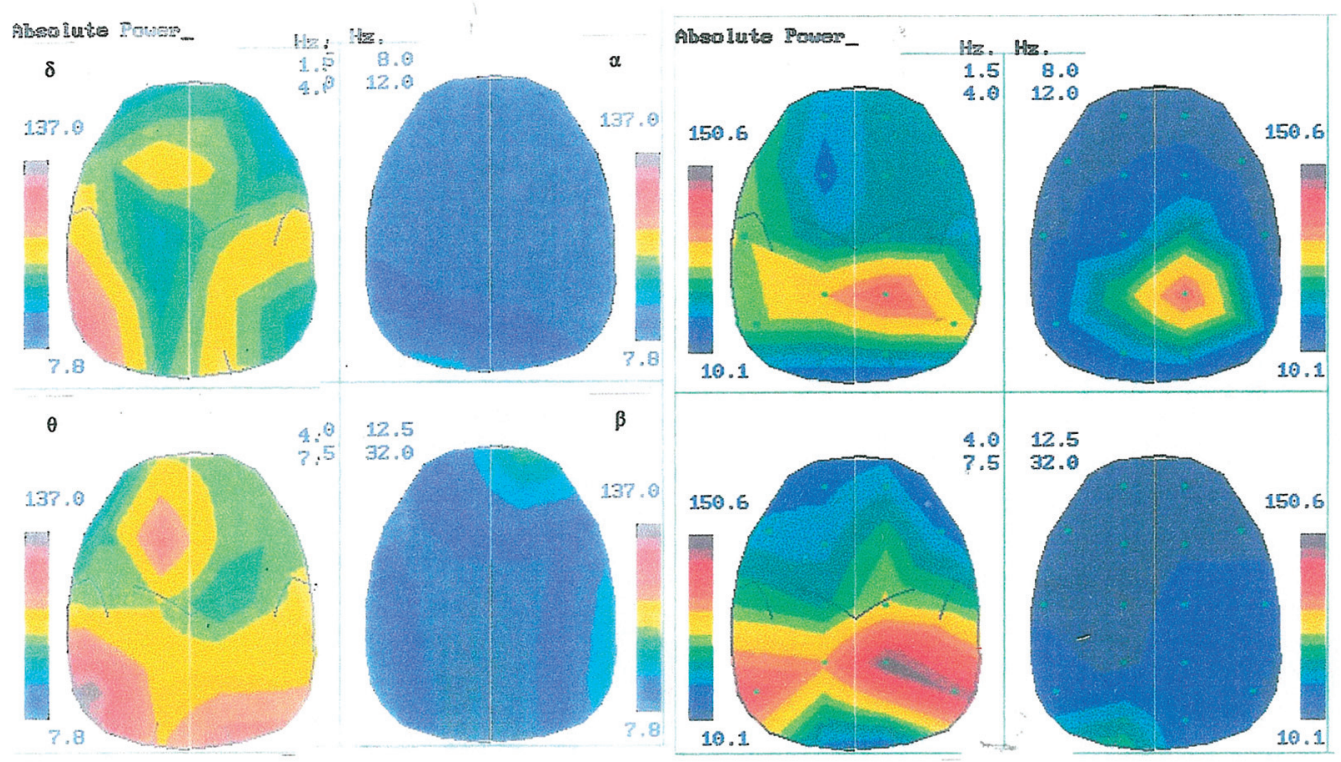

(a)

(b) 
FIGURE 3. Listening tests for the right and left ear of Lena during (above) and after the therapy (below). Note. The spatial errors in the air conduction are indicated by blue stars and in the bone conduction by red crosses.
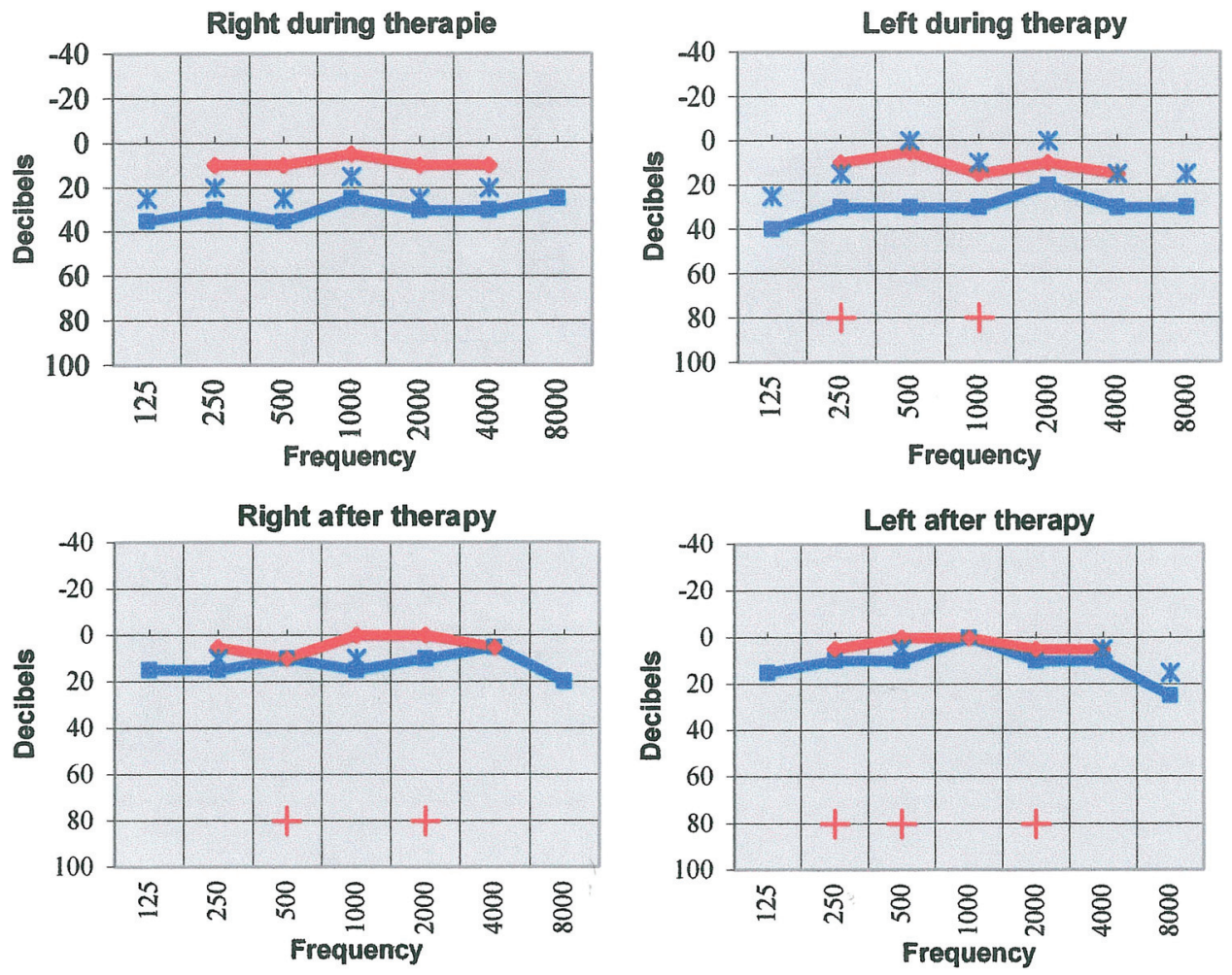

shows up both at low frequencies, representing the neural vegetative processes; at middle frequencies, thus in communication and the development of speech; and at high frequencies, representing thinking processes. The air conduction results (blue line) follow the same pattern but at a much lower level, indicating poor perception and communication with the outside world, thus little alertness. Such was also observed in the brain map, as indicated by almost the complete absence alpha activity all over the scalp. The many spatial errors indicate disorientation and confusion. The transfer of sounds by bone conduction in the skull causes the spatial errors from left to right or vice versa and are consistent with the observed absence of alpha activity in the brain map. Further treatments accelerated development, particularly in motor, speech, and coordination, and in the domain of consciousness to open up and interact with the outside world.

Many listening tests and brain maps had recorded changes in the development. The

latest listening tests at age 7 demonstrated the great improvement. The small gap now observed between bone and air conduction illustrates the large improvements. This signifies more harmony between the inner and outer world. The balance between low and high frequencies is more positive also. This enables a better coordination between body and spiritual processes, thus a more structural functioning. The air conduction test shows further improved reproduction in the essential speech domain between 1000 and $3000 \mathrm{~Hz}$, particularly of the left ear. The spatial errors also diminished. Indeed, her speech has developed very well. The latest brain map confirmed the improvements (Figure 2b): There was now alpha activity of 8 to $9 \mathrm{~Hz}$, maximal at P4. Also the oddball AEP curves showed considerable improvements. A normal N100 impulse amplitude is measured under the condition of focused alertness. Normal automatic stimulus discrimination is also observed at N200 as well as normal P300 latency time 
and amplitude, which signifies normal, controlled meaningful stimulus processing.

The parents also contributed by their extraordinary perseverance and discipline during the long-lasting therapy. Lena is now at the threshold of a total development consistent with that of other children. Apart from her physical development she seems only 1 year behind as compared to other children of her age in the field of learning abilities and social attitudes as observed by the clinician, by the family doctor and by nurses in the kindergarden.

\section{Case 2: Johanna}

Johanna had a history of expressive development aphasia and speech development retardation. She exhibited psychoneurological dysfunction, extensive retarded development, and general disturbance of obsevation; showed no concentration and extensive speech problems; lived in her own world; and showed no interest in the outside world.

After an early birth (35th week) by caesarian section, her weight was $2.424 \mathrm{~g}$, and she had many severe problems. For instance, a magnetic resonance imaging (MRI) scan showed agenesis of the corpus callosum and a CT scan of the skull showed atrophy in the right cerebral and left temporal lobes with large cisterna magna. Other problems occurred afterward, such as cyanosis and hypothonia. From age 4 onward, several EEGs indicated left hemispheric epileptic activity and hypersynchrony. Different medications had been prescribed, such as Opsolat (Sultiam), Sabril, and Frisum, with fluctuating results. The allover pattern had been interpreted in various medical reports as suggestive for the Landau-Klefner syndrome, characterized by aphasia and bioelectric epileptic paroxysms.

Johanna was provided with therapy for about 2 years, with 37 cycles in total. On February 28, 1996, when she was 5 years old, a first listening test was administered. It showed wild fluctuations and was rather chaotic. In that test the gap between the high bone conduction and the air conduction results as well as the closed selectivity, signified that she was living in her own world, separated from the others. The very weak, irregular, and low air conduction results indicated a distorted perception in all senses, amplified by the closed selectivity. This was particularly the case at low frequencies, up to $1000 \mathrm{~Hz}$, causing a poor vestibule function. As a result her concentration was very weak and she exhibited severe speech problems.

The first EEG-brain maps conducted on July 29, 1996, confirmed the early MRI, $\mathrm{CT}$, and EEG measurements. The large $\delta$ activity $(1.5-4.0 \mathrm{~Hz})$ with high-power left hemispherical with maximum at P3 and $\mathrm{O} 1$ was consistent with an epileptically disturbed trajectory.

The middle latency AEPs (MLAEPs), evoked by pure tones, are plotted in Figure 4. The results shown in Figure 4a, also obtained on July 29, 1996, presents the response after left and right stimulation. The left response was larger than that on the right, consistent with language development disorder. Given are T4 (right temporal; solid line) and T3 (left temporal; broken line) MLAEPs to enable extraction of the Mason asymmetry index, being T4/T3$\mathrm{T} 3 / \mathrm{T} 4=-0.12$. Normal would be $0.5+0.5$, whereas language development disorder would score $-0.25+0.5$. This indicated that Johanna's index was closer to pathology than normal.

Further listening therapy followed for about 2 years with 19 extra cycles. The latest listening test, at age 8 years 6 months, shows a remarkable improvement with rather regular bone and air conduction curves close together, on both left and right sides. The MLAEP amplitudes derived from the EEG data on August 5, 1998, showed a normal pattern with a rather symmetrical response in the right temporal side and the left one (see Figure 4b).

Notwithstanding her slow development and in spite of heavy medications, Johanna has experienced remarkable improvement that has lasted for many years after therapy terminated, as measured by a test at age 12 . She is in contact with her surroundings and participates in activities. She speaks better, has good eye contact, plans a lot, and wants 
FIGURE 4. Middle latency auditory evoked potentials of Johanna after the beginning (a) and after the therapy (b). Note. The strong asymmetry left-right at the beginning has disappeared after the therapy. In Figure 4a the solid line represents the T4 (right temporal) complex and the broken line the T3 (left temporal) complex after stimulation the left and the right ear. Note that the negative voltage, in microvolt, is plotted upward. In Figure $4 \mathrm{~b}$ the upper curve represents the T4 complex, the middle one the T3 complex and the lower one the center position $\mathrm{Cz}$, all with respect to the same reference. The vertical scale is relative, because it concerns three curves with each positive and negative electrical potentials, but with the same adjustment as in Figure $4 a$.

AUDITORY EUDKED POTENTIALS

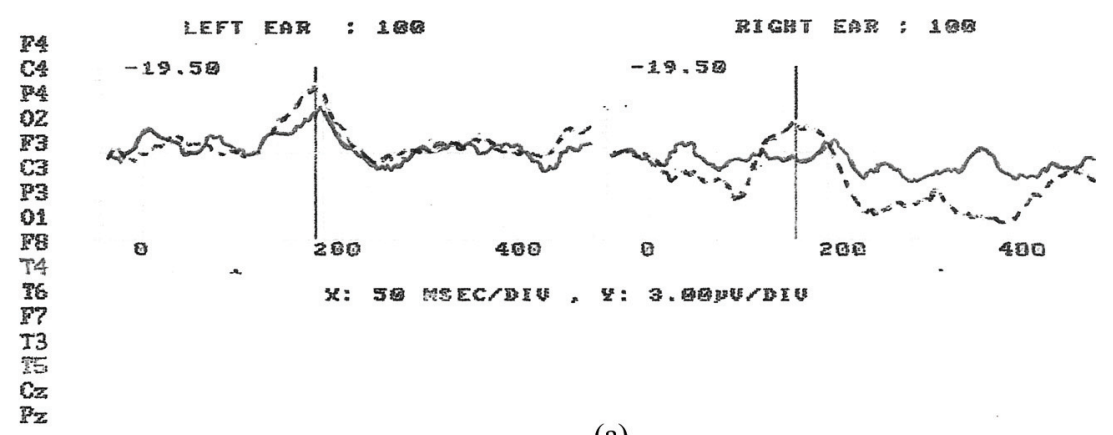

(a)

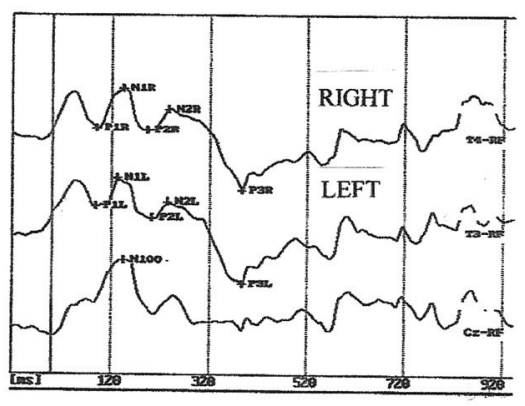

(b)

to improve her knowledge. Also her fine motor control has improved.

\section{Case 3: Francis}

Francis was born with deficits that resulted in a shortage of oxygen, was autistic, did not talk (analyzed at the University of Louvain), was hyperactive and very aggressive, Had psycho-motor retardation, and exhibiting retardation in language development. Francis was provided with an intensive listening therapy for $1 \frac{1}{2}$ years. The first somewhat reliable listening test was obtained on July 12, 1995, and exhibited a large difference between bone and air conduction tests with strong and chaotic irregularities, consistent with the problems of Francis (see Figure 5). Eighteen months later, on January 10, 1997, the test showed large improvements, although the bone conduction results were still too high.

In Figure 6 the EEG-brain mapping data are compared before and after the therapy. The brain map before the therapy (Figure 6) shows an asymmetry in the amplitudes of the MLAEPs with dominance of the left hemisphere. With a stimulus to the left ear the MLAEP response left temporally (T-complex, broken line) was larger than right (solid line), as can be seen typically in language development disorders. Normally this test shows a symmetric T-complex or a 
FIGURE 5. The Listening tests of Francis at the beginning (above) and after the therapy (below).
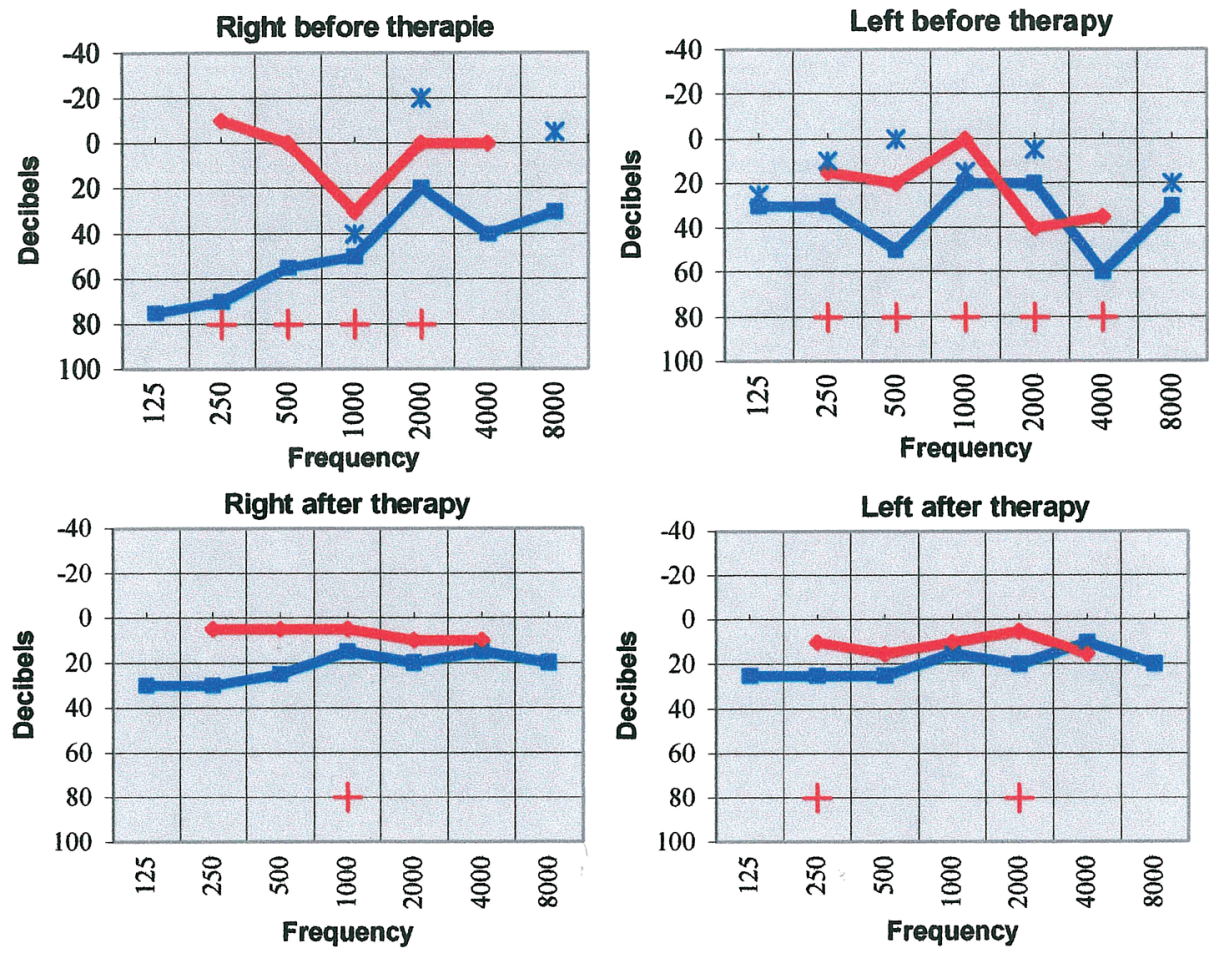

reversed asymmetry (response higher right temporally than left). This imbalance was restored after the therapy, with even a stronger response on the right than the left. The Mason asymmetry index (see Case 2) before the therapy was -0.64 , thus compatible with a language development disorder. After the therapy this index was restored to normal.

Francis has experienced great progress in the $1 \frac{1}{2}$ years of intensive listening therapy. The boy has become quieter, with more concentration and better speech. He has become markedly alert and interacts with the outside world. These changes have been observed by the clinician and parents and have been documented by his progress in speech and communication with the environment, particularly at school.

\section{Case 4: Ambroise}

Ambroise, a boy, showed extensive retardation in general development, was born 1 month too early, and after $1 \frac{1 / 2}{2}$ months suffered from epileptic attacks; he had fallen on his head at age 2 and it resulted in brain

damage, particularly cerebral hemorrhage, affecting predominantly the frontal and left-temporal hemispheres, and he exhibited behavior that was chaotic, as he was difficult to manage.

Because of his age of 2, no listening tests could be obtained. The first EEG-brain mapping taken at the start of the therapy shows a very slow base rhythm of 2 to $5 \mathrm{c} / \mathrm{sec}$ sec in the $\delta$ domain, with a maximum in the posterior region and with a certain dominance at the left side, at F3, T3, and T5 (see Figure 7).

The oddball paradigm AEP lacks the essential N100, N200, and P300 structures, indicating serious auditory perception troubles. After the first cycle of 14 days, several 6-day cycles about 6 weeks apart continued for the rest of 2003. He reacted very well and showed important improvements, particularly in the movements of his legs, arms, and hands. He even started babbling. The improvement is also seen in the EEG taken at the end of 2003 (see Figure 7b). There appeared to be more harmony in the $\delta$ domain. There are no epileptic tendencies anymore, and the drug Epitomax was being 
FIGURE 6. Middle latency auditory evoked potentials and brain maps of Francis before (a) and after the therapy (b). Note. The solid line represents the T4 complex and the broken line the T3 complex. The T complexes are measured as a sum of the P100, N100, P200, and N200 intensities, shortly indicated by P1-N1-P2-N2. Note that in the brain map the blue color represents electrical negative voltage, thus high brain activity and the red color electrical positive voltage, thus low brain activity, as opposed to the color indications in Figures 2 and 7.

\section{BRAIN MAPPING IN CENTRAL AUDITORY DYSFUNCTION}

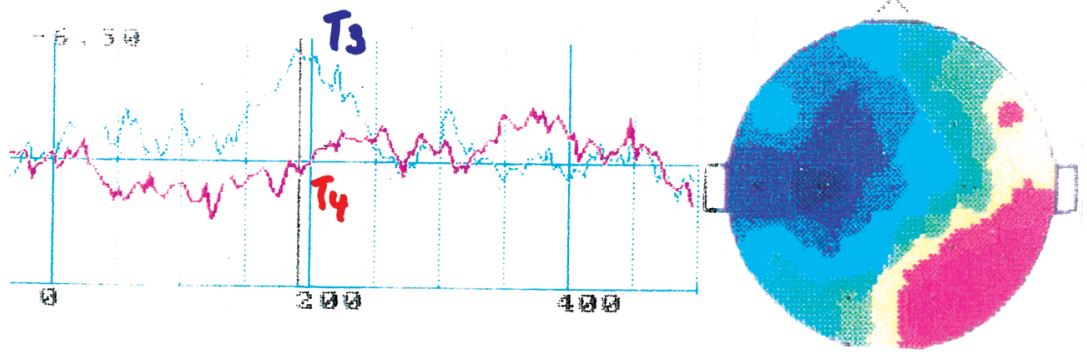

(a)

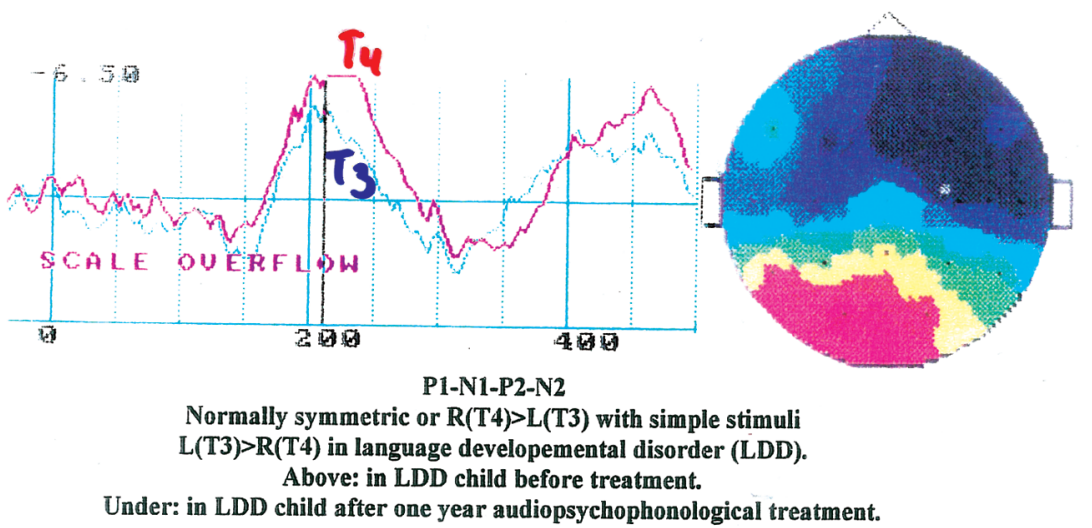

(b)

FIGURE 7. Brain maps of Ambroise before (a) and after the therapy (b). Note. There are different scales in the maps $a$ and $b$, being a factor of 2.8 larger in case $b$, indicate relative brain activity (deduced from the electrode voltages) with red high and blue low brain activity.

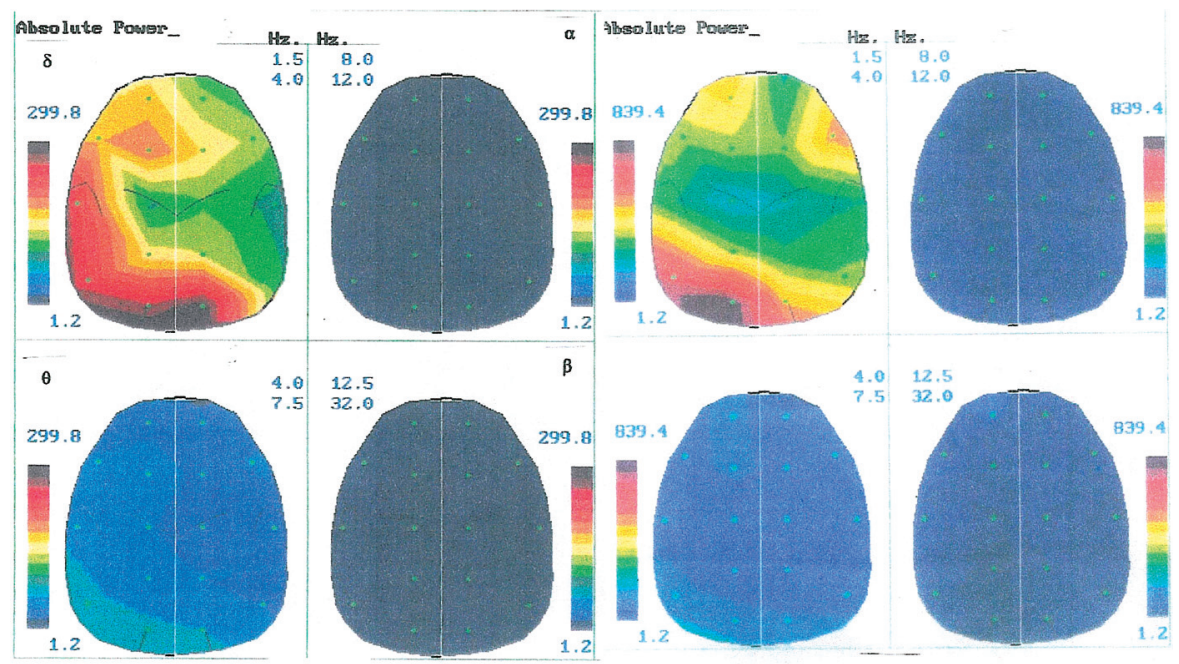

(a)

(b) 
diminished. The oddball paradigm AEPs now weakly showed the responses of N200 (automatic discrimination of stimuli) and P300 (conscious perceptual discrimination).

Ambroise has experienced great progress in only 6 months of listening therapy. It was recommended that intensive therapy continued for about 6 to 9 weeks.

\section{DISCUSSION}

These four participants are only a small selection out of many thousands of clients treated successfully. Earlier treatments are documented less well, because the EEGbrain mapping has been operational at the Atlantis and MBL institutes only during the last decennium. Numerous brain maps and letters by parents, schools, and medical doctors support the remarkable improvements obtained over a wide spectrum of development disorders. These data suggest that the results reported here are not isolated incidents but rather common in numerous cases.

It can be concluded that the listening tests were consistent with the EEG-brain-map data, thus independently correlating the results of those tests. In most cases attention and concentration problems were observed during the listening test by the high and often descending bone conduction results at low frequencies with respect to the air conduction test results. This correlated strongly with large $\delta$ activity frontal and prefrontal and with the absence or weak $\alpha$ activity, mostly occipital, in the corresponding brain maps and with weak N200 and P300 amplitudes in the oddball paradigm AEPs, both indicating little alertness. After the listening therapy those aspects were improved in the listening tests, as well as in the brain maps and AEPs. Generally a correlation was also observed in the case of language disorders between the diminished sensitivity in the middle frequency region of about 1000 to $3000 \mathrm{~Hz}$ in the listening tests and the diminished activity at the temporal lobes in the brain maps. Therefore, listening tests can be used as reliable evidence to support the results of the listening therapy.
The four cases reported here concern very severe disorders and required relatively long and intensive treatments. Less severe but rather uncomfortable disabilities in communication, concentration, dyslexia, speech, and language have been treated successfully with a year of listening therapy. It is essential that the therapy be administered by welltrained consultants in a location with the appropriate equipment because of the specific adjustments that need to be made in the special electronic apparatus and because continuous observation of the reactions of the clients is needed.

\section{REFERENCES}

Madaule, P. (1994). When listening comes alive. Ontario, Canada: Moulin.

Mason, S. M., \& Mellor, D. H. (1984). Middle latency and late cortical evoked potentials in children with speech and language disorders. Electroencephalography and Clinical Neurophysiology, 59, 297-309.

Merzenich, M. M., Jenkins, W. M., Johnston, P., Schreiner, C., Miller, S. L., \& Tallal, P. (1996). Temporal processing deficits of language-learning impaired children ameliorated by training. Science, 271, 77-81.

Pinkerton, F., Watson, D. R., \& McClelland, R. J. (1989). A neurophysiological study of children with reading, writing and spelling difficulties. Developmental Medicine and Child Neurology, 31, 569-581.

Schydlo, R. (2002), Kinderpsychiatrische Stõrungen als Folge auditiver Wahrnehmungsstõrungen. (Child psychiatric distortions caused by auditory perception disorders.) Unpublished report, Düsseldorf, Germany.

Tallal, P., Miller, S. L., Bedi, G., Byma, G., Wang, X., Nagarajan, S. S., et al. (1996). Language comprehension in language-learning impaired children improved with acoustically modified speech. Science, 271, 81-84.

Tallal, P., Miller, S., \& Fitch, R. H. (1993). Neurobiological basis of speech: a case for the pre-eminence of temporal processing. Annals of the New York Academy of Science, 682, 27-47.

Tomatis, A. A. (1972). Education et dyslexie [Education and dyslexia]. In Coll. Sciences de l'Education. Fribourg, France: AIAPP Editions.

Tomatis, A. A. (1981). La nuit uterine [The uterine night]. Paris: Verlag Editions Stock.

Tomatis, A. A. (1989). Vers l'ecoute humaine [Toward human listening] (D. Zimmermann, Ed.) Paris: ESF. 
Tomatis, A. A. (1991). The conscious ear (B. M. Thompson, Ed.). Barrytown, NY: Station Hill Press.

Van den Bergh, W. (1998). Die neurologische Basis der audio-psycho-phonologischen Therapie bei Sprachentwicklungsstõrungen und Legasthenie.
(Neurological basis of APP treatment of disturbances in language acquisition and dyslexia.) In: Jozef and Marie-Jeanne Vervoort: Wissen Sie, warum Sie zwei Ohren haben? (Do you know why you do have two ears?) Atlantis-vzw Institute, Sint-Truiden, Belgium, pp. 74-81. 